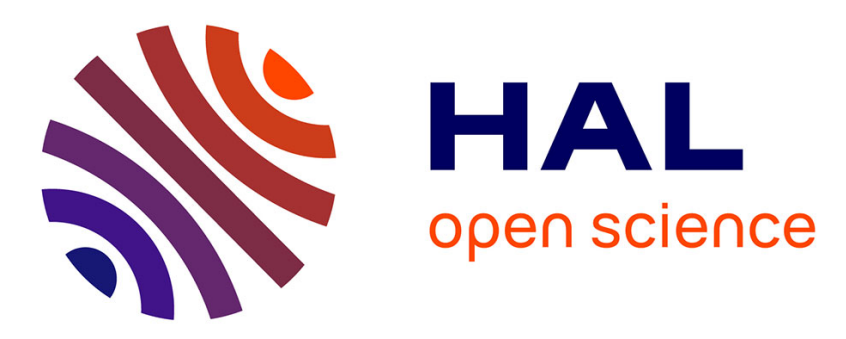

\title{
Conductivité électrique de solutions de surfactants dans les hydrocarbures
}

\author{
A. Denat, B. Gosse, J.P. Gosse
}

\section{To cite this version:}

A. Denat, B. Gosse, J.P. Gosse. Conductivité électrique de solutions de surfactants dans les hydrocarbures. Revue de Physique Appliquée, 1981, 16 (12), pp.673-678. 10.1051/rphysap:019810016012067300 . jpa-00244961

\section{HAL Id: jpa-00244961 https://hal.science/jpa-00244961}

Submitted on 1 Jan 1981

HAL is a multi-disciplinary open access archive for the deposit and dissemination of scientific research documents, whether they are published or not. The documents may come from teaching and research institutions in France or abroad, or from public or private research centers.
L'archive ouverte pluridisciplinaire HAL, est destinée au dépôt et à la diffusion de documents scientifiques de niveau recherche, publiés ou non, émanant des établissements d'enseignement et de recherche français ou étrangers, des laboratoires publics ou privés. 


\title{
Conductivité électrique de solutions de surfactants dans les hydrocarbures
}

\author{
A. Denat, B. Gosse et J. P. Gosse \\ Laboratoire d'Electrostatique, avenue des Martyrs, B.P. 166X, 38042 Grenoble Cedex, France
}

(Reçu le 12 juin 1981, accepté le 18 septembre 1981)

\begin{abstract}
Résumé. - La conductivité des solutions d'un surfactant ionique (Aerosol OT) dans des hydrocarbures est mesurée en faible tension alternative et en impulsion de tension. L'influence de différents paramètres tels que la tension appliquée, la distance entre les électrodes est étudiée pour des concentrations en surfactant variant de $10^{-5} \mathrm{Ml}^{-1}$ à $10^{-2} \mathrm{Ml}^{-1}$. L'ensemble des résultats est interprété par l'existence d'agrégats $\mathrm{S}$ dans la solution se dissociant suivant la réaction équilibrée globale $2 \mathrm{~S} \rightleftarrows \mathrm{S}^{+}+\mathrm{S}^{-}$. L'étape la plus lente du schéma réactionnel est $\mathrm{AOT} \rightleftarrows \mathrm{Na}^{+}+\mathrm{B}^{-}$, elle détermine le comportement de la solution mise hors d'équilibre par le champ électrique appliqué.
\end{abstract}

\begin{abstract}
The conductivities of solutions of an ionic surfactant in hydrocarbons were investigated experimentally with small AC voltages and with voltage wave-forms, over a wide range of applied voltages and various electrode spacings, the concentration of the surfactant ranging from $10^{-5}$ to $10^{-2} \mathrm{Ml}^{-1}$. The observed behaviour is explained by the existence of aggregates $S$ in the solution, even at very low concentrations, $S$ dissociating according to the overall process $2 \mathrm{~S} \rightleftarrows \mathrm{S}^{+}+\mathrm{S}^{-}$. The overall rate of reaction is controlled by the step AOT $\rightleftarrows \mathrm{Na}^{+}+\mathrm{B}^{-}$ which determines the conductivity of the solution put out of equilibrium by a dc field.
\end{abstract}

1. Introduction. - Les différentes études des solutions de surfactant ionique dans un solvant non polaire ont été effectuées selon deux préoccupations essentielles et sans rapport entre elles :

1. Pour obtenir une solution de faible résistivité $\left(<10^{10} \Omega . \mathrm{m}\right)$ par dissolution d'un produit en très faible quantité dans les carburants $\left(C \leqslant 10^{-5} \mathrm{Ml}^{-1}\right)$, ce qui diminue le temps de relaxation des charges électriques formées lors de l'écoulement de l'hydrocarbure dans des tubes ou des filtres métalliques et donc les risques d'incendie ou d'explosion [1]. La préoccupation dans ce cas était l'obtention d'une solution la plus conductrice possible pour une concentration en surfactant donnée.

2. Pour des problèmes liés à la récupération assistée du pétrole aux détergents, il a été étudié la structure des agrégats que forment les surfactants en solution concentrée $\left(>10^{-3} \mathrm{Ml}^{-1}\right)$ dans un hydrocarbure. Les résultats obtenus par des méthodes physiques diverses ont été confrontés aux études physicochimiques des solutions micellaires [2]

Il y a eu peu d'études de la conduction électrique de ces solutions sous champs électriques élevés $[3,4]$; nous décrivons dans ce papier nos observations portant sur des solutions de di-2-éthylhexyl sulfosuccinate de sodium (Aerosol OT) dans le benzène et le cyclohexane, sur les variations de la conductivité de ces solutions avec la concentration en Aerosol OT, le comportement sous tension continue et l'influence du champ électrique sur la dissociation de l'électrolyte. Ces résultats seront comparés aux études antérieures sur les solutions d'Aerosol OT dans un hydrocarbure, ainsi qu'à nos propres études sur le comportement sous champ électrique des solutions d'un électrolyte du type ionophore (picrate de triisoamylammonium) dans un hydrocarbure [5].

2. Partie expérimentale. - Le surfactant ionique étudié est l'Aerosol OT de provenance Merck qui a été soigneusement purifié et desséché. Les solvants utilisés sont le benzène et le cyclohexane (produits Merck pour spectroscopie).

L'électrolyte a été soigneusement purifié et ensuite séché sous vide pendant plusieurs jours. La teneur en eau des solutions ainsi préparées ne varie pratiquement pas avec la concentration en électrolyte et vaut $5 \times 10^{-4} \mathrm{Ml}^{-1}$.

La conductivité de ces solutions à diverses concentrations a été mesurée dans des cellules de conductimétrie à l'aide d'un pont de mesures en faible tension alternative et de fréquence variable $(10 \mathrm{~Hz}$ à $100 \mathrm{kHz})$ (Pont Général Radio 1621). Le courant traversant ces mêmes solutions a été étudié lors de l'application 
d'un échelon de tension variable de 0 à $20 \mathrm{kV}$ entre deux électrodes en acier inoxydable distantes de 0,3 à $3,25 \mathrm{~mm}$; la tension apparaissant aux bornes d'une résistance de faible valeur placée en série avec la cellule est enregistrée à l'oscilloscope, le courant de conduction peut être mesurée $100 \mu$ s après la mise sous tension.

3. Résultats expérimentaux. - 3.1 CONDUCTIVITÉ DES SOLUTIONS D'AEROSOL OT DANS LES HYDROCARBURES MESURÉE SOUS TENSION ALTERNATIVE. - Les variations de la conductibilité équivalente $\Lambda=10^{3} \sigma / C$ (où $\sigma$ est la conductibilité de la solution et $C$ la concentration en $\mathrm{Ml}^{-1}$ ) de l'Aerosol OT dans le benzène en fonction de la concentration $C$ sont représentées sur la figure 1 , courbe $a$.

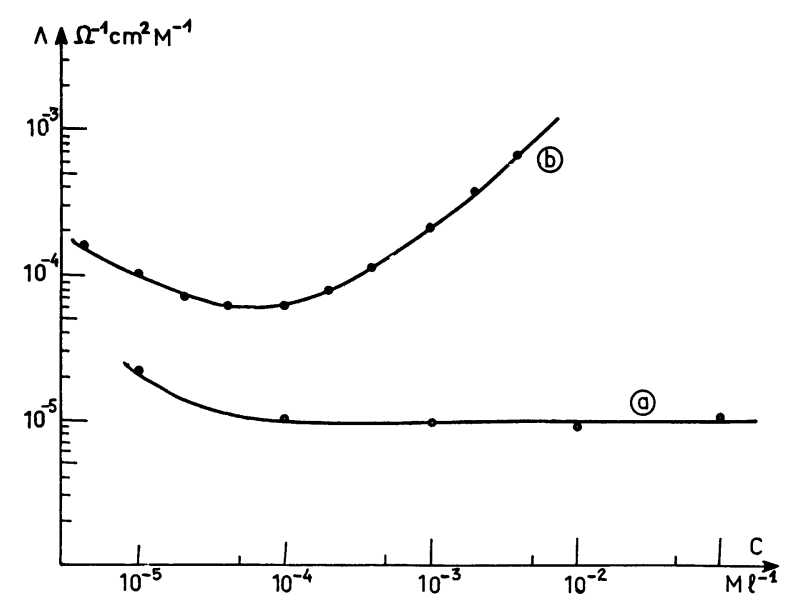

Fig. 1. - Conductivité équivalente de solutions d'Aerosol OT (courbe $a$ ) et de picrate de tétrabutylammonium (TIAP) (courbe $b$ ) dans le benzène en fonction de la concentration. Tension de mesure $10 \mathrm{~V}$ crête à crête.

[Concentration dependence of the equivalent conductivity of Aerosol OT (curve $a$ ) and of tetrabutylammonium picrate (curve $b$ ).]

Dans la gamme de concentration de $5 \times 10^{-5}$ à $10^{-1} \mathrm{Ml}^{-1}, \Lambda$ est indépendante de $C$. Nous avons retrouvé le même résultat pour des solutions d'Aerosol OT dans le cyclohexane. La courbe $b$ de la figure 1 représente les variations $\Lambda(C)$ de solutions de picrate de tétrabutylammonium (TBAP) dans le benzène. Il apparaît une différence très nette de comportement de ces solutions, ce qui avait déjà été observé $[1,2,6]$.

Dans le cas du TBAP, la variation $\Lambda(C)$ est bien comprise depuis les travaux de Fuoss et Kraus [7]; les forces électriques entre les ions sont très fortes en milieu hydrocarbure et il apparaît une association ionique qui dépend de la concentration en électrolyte selon le processus ions-paires d'ions-triples ions-quadruplets...

Dans le cas des solutions d'Aerosol OT, la valeur de $\Lambda$ ne dépend pratiquement pas de la concentration bien que son coefficient de dissociation apparent $\alpha$ soit très faible (dans le benzène $\alpha \simeq 10^{-7}$ ) et que cet électrolyte dissout dans un solvant polaire soit entièrement dissocié. Ceci implique l'existence d'une agrégation indépendante de la concentration; on peut supposer l'existence d'un équilibre

$$
m \mathrm{AOT} \rightleftarrows(\mathrm{AOT})_{m}=\mathrm{S},
$$

$S$ étant un agrégat ; Eicke [8] a proposé la formation de trimères pour interpréter ses mesures de moment dipolaire de l'Aerosol OT dans les hydrocarbures.

Appelons $(\mathrm{AOT})_{\mathrm{th}}$ la concentration théorique en AOT définie comme le nombre de moles dissoutes par unité de volume, (AOT) la concentration en molécules d'AOT non associées et (S) la concentration en agrégat; on a la relation :

$$
(\mathrm{AOT})_{\mathrm{th}}=(\mathrm{AOT})+m(\mathrm{~S}) \text {. }
$$

Supposons que la conductibilité de la solution résulte d'un équilibre de dissociation du type $2 \mathrm{~S} \rightleftarrows \mathrm{S}^{+}+\mathrm{S}^{-}$. Les porteurs sont monovalents : dans le cas de microémulsions où les porteurs ont une taille encore plus importante, il a été prouvé qu'ils portent une seule charge électronique [9]. Les agrégats $\mathrm{S}^{+}$et $\mathrm{S}^{-}$étant quasiment identiques ont la même mobilité $k$. La conductibilité $\sigma$ de la solution s'écrit alors $\sigma=2 k e\left(\mathbf{S}^{+}\right)$avec $\left(\mathrm{S}^{+}\right)=\left(\mathrm{S}^{-}\right)$.

A l'équilibre thermodynamique, on peut écrire $\left(\mathrm{S}^{+}\right)\left(\mathrm{S}^{-}\right) /(\mathrm{S})^{2}=K$ soit $\sigma=2 k e(\mathrm{~S}) \sqrt{K}$. $\Lambda$ s'écrit :

$$
\Lambda=10^{3} \sigma /(\mathrm{AOT})_{\mathrm{th}}
$$

soit

$$
\Lambda=10^{3} \times 2 k e \sqrt{K}(\mathrm{~S}) /((\mathrm{AOT})+m(\mathrm{~S})) .
$$

Pour obtenir $\Lambda$ indépendant de la concentration théorique (AOT), on est amené à supposer $(\mathrm{AOT}) \ll m(\mathrm{~S})$, c'est-à-dire que toutes les molécules d'Aerosol OT sont incluses dans des agrégats. On

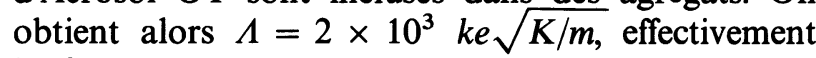
indépendant de $(C)$.

En conclusion, les variations $\Lambda(C)$ de solutions de surfactant dans les hydrocarbures peuvent s'expliquer par la formation d'agrégats qui mettraient en jeu toutes les molécules d'Aerosol OT et qui se dissocieraient suivant un schéma réactionnel, peut-être complexe mais dont la réaction globale serait $2 \mathrm{~S} \rightleftarrows \mathrm{S}^{+}+\mathrm{S}^{-}$. Ces hypothèses seront confirmées par l'étude de la conductibilité de ces solutions sous champs intenses.

3.2 ACTION D'UN CHAMP ÉLECTRIQUE CONTINU. Un exemple caractéristique de courbe courant en fonction du temps relevée lors de l'application d'un échelon de tension à une solution d'Aerosol OT dans le cyclohexane est donné sur la figure 2. Le courant qui vaut $I_{0}$ à l'instant initial de la mise sous tension, décroît pendant un temps $t_{0}$, il prend alors une valeur constante $I_{\mathrm{p}}$. Le courant en fait continue de décroître après le temps $t_{0}$, mais très faiblement (après $50 t_{0}$ 

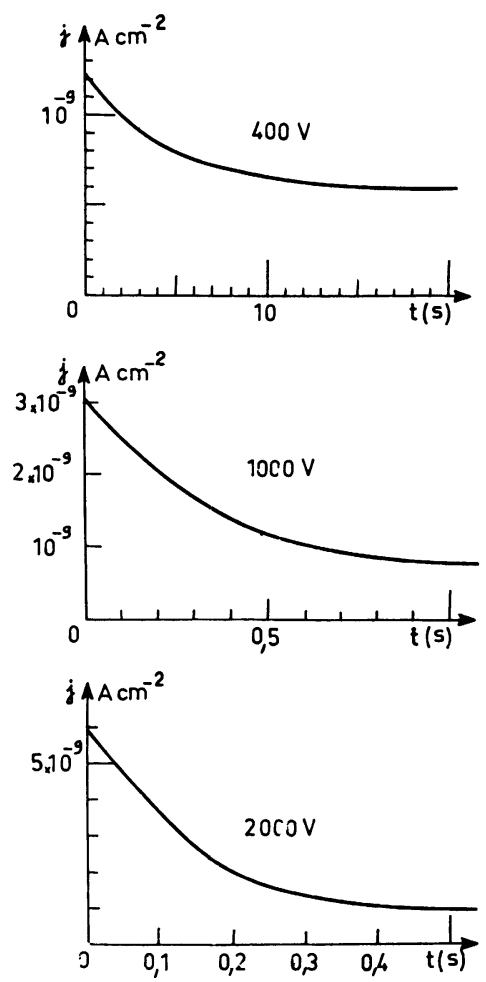

Fig. 2. - Courant transitoire dans une solution d'Aerosol OT dans le cyclohexane. Concentration $10^{-4} \mathrm{Ml}^{-1}$; tension : $2000 \mathrm{~V}$; distance entre les électrodes : $1,55 \mathrm{~mm}$.

[Typical current transient observed with voltage wave-form in cyclohexane, the Aerosol OT concentration was $10^{-4} \mathrm{Ml}^{-1}$, voltage $2000 \mathrm{~V}$, cell thickness $1.55 \mathrm{~mm}$.]

il a baissé de $10 \%$ environ). Nous avons vérifié que le courant $I_{0}$ est égal au courant déduit de la mesure de la conductance de la cellule au moyen d'un pont d'impédance. Il varie bien comme $V$ et comme $d^{-1}$ pour l'ensemble des mesures, le champ électrique variant entre 0,1 et $200 \mathrm{kV} \mathrm{cm}{ }^{-1}$, la distance entre les électrodes entre 0,3 et $3,25 \mathrm{~mm}$, la concentration en Aerosol OT entre $10^{-5}$ et $10^{-2} \mathrm{Ml}^{-1}$.

La densité de courant de palier $I_{\mathrm{p}}$ est donnée sur la figure 3 en fonction de la tension appliquée pour une concentration en Aerosol OT de $10^{-4} \mathrm{Ml}^{-1}$ et une distance de $1,52 \mathrm{~mm}$. Sur cette courbe $j(V)$ on distingue une première partie dans laquelle le courant est proportionnel à la tension (régime ohmique). Pour une tension supérieure à $V_{\mathrm{s}}$; la densité de courant croît moins vite que la tension, on a conduction à saturation. Novotny et Hopper [4] dans une étude de l'Aerosol OT dissous dans du xylène ont obtenu des résultats similaires mais ils ont surtout analysé les phénomènes transitoires survenant après un renversement de tension sur la cellule.

Ces résultats sont caractéristiques d'un régime de conduction régie par un équilibre de dissociationrecombinaison entre des espèces neutres et des ions; ce type de conduction a été décrit par J. J. Thomson [10] dans le cas des gaz ionisés, son modèle a été appliqué par plusieurs auteurs au cas des liquides [11, 12].

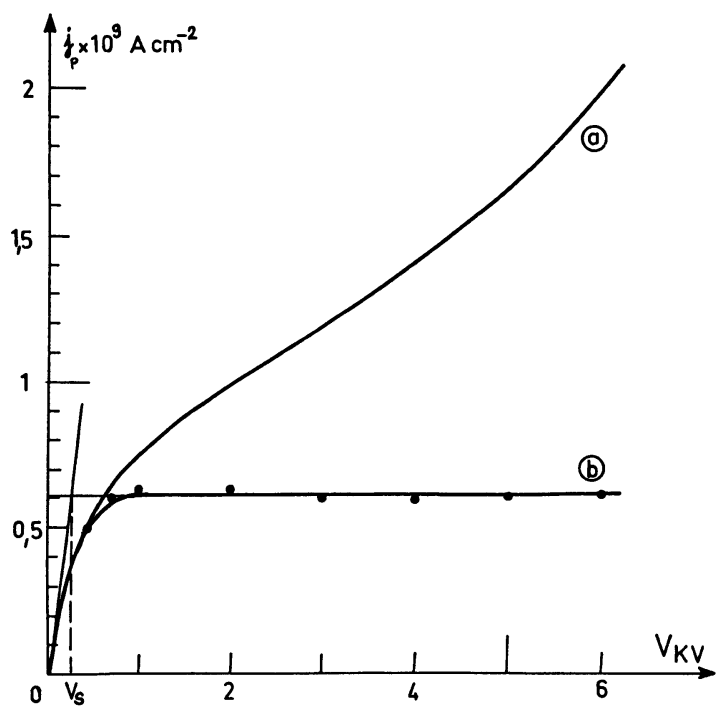

Fig. 3. - Caractéristique $j(V)$ d'une solution d'Aerosol OT dans le cyclohexane (courbe $a$ ); concentration : $10^{-4} \mathrm{Ml}^{-1}$; distance entre les électrodes : $1,52 \mathrm{~mm}$; caractéristique calculée $j / F(b)$ en fonction de la tension appliquée (courbe $b$ ).

[Current density $j$-voltage $V$ characteristic for Aerosol OT in cyclohexane; the Aerosol OT concentration was $10^{-4} \mathrm{Ml}^{-1}$, cell thickness $1.52 \mathrm{~mm}$. $j$ curve $(a)$; ratio $j / F(b)$ curve $(b)$.]

L'apparition d'ions dans un liquide en l'absence de causes extérieures est attribuée selon le modèle de Bjerrum [13] à une réaction équilibrée décomposable en deux étapes successives : $\mathrm{AB} \underset{(1)}{\rightleftarrows} \mathrm{A}^{+} \mathrm{B}^{-} \underset{(2)}{\rightleftarrows} \mathrm{A}^{+}+\mathrm{B}^{-}$; (1)

la réaction 2 qui correspond au mécanisme lent, impose sa cinétique à l'équilibre résultant. Dans le cas de l'Aerosol OT entièrement dissocié dans les solvants polaires (AOT est dit ionophore), la réaction équilibrée se réduit à la réaction 2. L'apparition d'ions dans le liquide résulte donc d'une part d'une réaction de dissociation $\mathrm{A}^{+} \mathrm{B}^{-} \rightarrow \mathrm{A}^{+}+\mathrm{B}^{-}$avec une constante de vitesse $k_{\mathrm{D}}$ et une réaction de recombinaison $\mathrm{A}^{+}+\mathrm{B}^{-} \rightarrow \mathrm{A}^{+} \mathrm{B}^{-}$avec une constante de vitesse $k_{\mathrm{R}}$; $k_{\mathrm{R}}$ vaut d'après Langevin $k_{\mathrm{R}}=e\left(K^{+}+K^{-}\right) / \varepsilon, K_{+}$ et $K_{-}$sont les mobilités des ions positifs et négatifs, $\varepsilon$ la permittivité diélectrique du liquide. Cette valeur est en bon accord avec différentes observations faites dans les liquides [14].

Selon ce modèle, la densité de courant stationnaire $j_{\mathrm{s}}$, devrait être constante aux tensions élevées et égale à $: j_{\mathrm{s}}=k_{\mathrm{D}}$, C.e.d. ce qui n'est pas observé dans nos essais. Nous avons attribué l'absence de plateau horizontal dans la courbe $j(V)$ à l'effet du champ électrique sur l'équilibre de dissociation. Dans ce cas, $j_{\mathrm{s}}$ s'écrit $j_{\mathrm{s}}(E)=k_{\mathrm{D}}(E)$, C.e.d. avec $k_{\mathrm{D}}(E)=k_{\mathrm{D}}(0) F(b)$ où

$$
F(b)=1+b+\frac{b^{2}}{3}+\frac{b^{3}}{18} \ldots \quad \text { et } \quad b=\frac{e^{3}|E|}{8 \pi \varepsilon k^{2} T^{2}}
$$

d'après le calcul théorique d'Onsager [15] vérifié expérimentalement avec d'autres électrolytes [16]. La courbe $b$ de la figure 3 a été obtenue en divisant 
la valeur du courant par le facteur $F(b)$, nous obtenons alors une valeur constante pour le rapport $j_{\mathrm{s}} / F(b)=j_{\mathrm{s}_{\mathrm{o}}}$ dont la signification est bien un courant de conduction à saturation. En effet, l'étude de la densité de courant $j_{\mathrm{s}_{0}}$ nous montre qu'elle varie proportionnellement à la concentration en Aerosol OT entre

$$
10^{-5} \text { et } 2 \times 10^{-3} \mathrm{Ml}^{-1} \quad \text { (Fig. 4). }
$$

Pour une concentration donnée $\left(10^{-4} \mathrm{Ml}^{-1}\right), j_{\mathrm{s}_{0}}$ varie comme $d^{0,8}$ (Fig. 5) et non comme $d$; cette anomalie n'a pas été expliquée.

D'autre part, selon le modèle de Thomson, la tension de saturation s'écrit

$$
V_{\mathrm{S}}=\left(k_{\mathrm{D}} C k_{\mathrm{R}}\right)^{1 / 2} d^{2} /\left(K_{\mathrm{p}}+K_{\mathrm{n}}\right)
$$

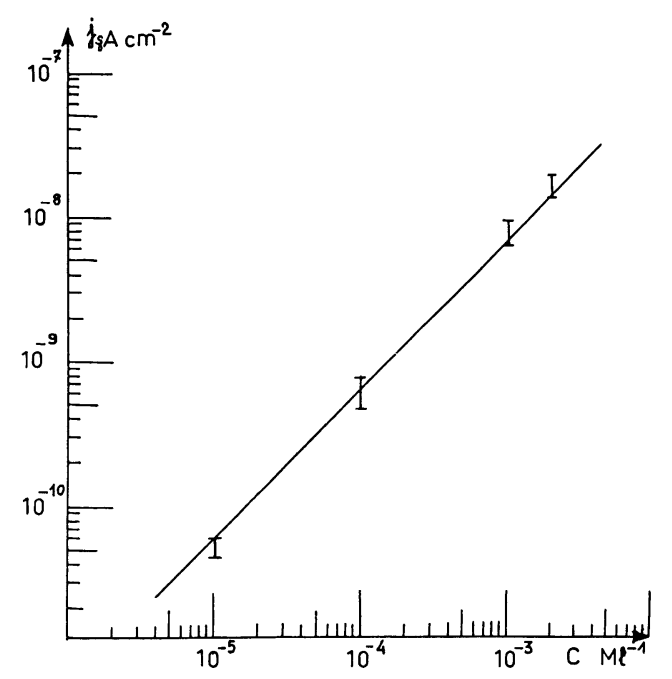

Fig. 4. - Variations de la densité du courant de saturation $j_{\mathrm{\varsigma}} / F(b)$ en fonction de la concentration en Aerosol OT dans le cyclohexane, distance entre les électrodes : 1,04 mm.

[Aerosol OT concentration dependence of the current of saturation $j_{\mathrm{s}} / F(b)$ in cyclohexane cell thickness $1.04 \mathrm{~mm}$.]

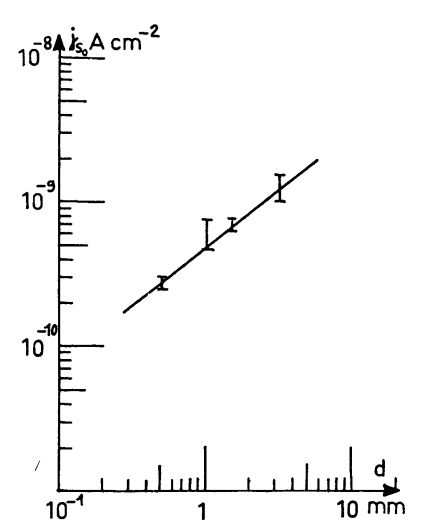

Fig. 5. - Variations de la densité du courant de saturation $j_{\mathrm{s}} / F(b)$ en fonction de la distance entre les électrodes, concentrations en $\mathrm{AOT}=10^{-4} \mathrm{Ml}^{-1}$.

[Electrode spacing dependence of the current of saturation $j_{\mathrm{s}} / F(b)$ in cyclohexane, concentration of Aerosol OT $=10^{-4} \mathrm{Ml}^{-1}$.] où $k_{\mathrm{D}}$ et $k_{\mathrm{R}}$ sont les constantes de vitesse de dissociation et de recombinaison, $C$ est la concentration en électrolyte et $K_{\mathrm{p}}, K_{\mathrm{n}}$ les mobilités des ions positifs et négatifs; $d$ est la distance entre les électrodes. Nous avons vérifié que dans nos essais $V_{\mathrm{S}}$ varie comme $d^{2}$ pour une concentration donnée en surfactant et que $V_{\mathrm{S}}$ varie comme $C^{1 / 2}$ pour une distance $d$ fixe.

\subsection{DéTERMINATION DE LA MOBILITÉ DES PORTEURS.} - L'étude du transitoire de conduction nous permet de déterminer la valeur de la mobilité moyenne des ions puisque le modèle de Thomson est vérifié dans nos solutions. Lorsque le nombre adimensionnel $C_{0}=q_{0} d^{2} / \varepsilon V$ est tel que $C_{0} \ll 1$, ce qui correspond à $V \gg V_{\mathrm{S}}$ pour une concentration en ions $q_{0}$ et une distance $d$ données, le courant est saturé et la distorsion du champ électrique par la charge d'espace est négligeable; la densité de courant en fonction du temps s'écrit :

$$
j(t)=\left(K_{\mathrm{p}} \mathrm{p}+K_{\mathrm{n}} \mathrm{n}\right) \frac{V}{d}\left(1-\frac{t}{T_{\mathrm{t}}}\right)
$$

si l'on néglige $j_{\mathrm{s}}$ (cas où $V \geqslant 5 V_{\mathrm{s}}$ ) et

$$
T_{\mathrm{t}}=\frac{2 d^{2}}{\left(K_{\mathrm{p}}+K_{\mathrm{n}}\right) V}=\frac{d^{2}}{K_{\mathrm{m}} V},
$$

$T_{\mathrm{t}}$ est le temps de transit des ions et $K_{\mathrm{m}}$ la mobilité moyenne. La courbe $I(t)$ de la figure 2 est caractéristique de cette équation puisque le courant décroît linéairement avec le temps. Nous avons vérifié que ce temps varie comme $d^{2}$ et comme $V^{-1}$; la mobilité moyenne $K_{\mathrm{m}}$ déduite de la mesure du temps de transit est indépendante de la concentration entre $10^{-5}$ et $2 \times 10^{-3} \mathrm{Ml}^{-1}$ d'Aerosol OT dans le cyclohexane et vaut $6 \times 10^{-5} \mathrm{~cm}^{2} \mathrm{~V}^{-1} \mathrm{~s}^{-1}$; ceci est à comparer à la valeur de la mobilité du picrate de triisoamylammonium (TIAP) dans le même solvant qui vaut $1,3 \times 10^{-4} \mathrm{~cm}^{2} \mathrm{~V}^{-1} \mathrm{~s}^{-1}$. Nous avons effectué des mesures en géométrie cylindrique puisqu'elles permettent de déterminer s'il existe une différence notable entre les mobilités des ions positifs et négatifs [17]; nous avons observé dans ce cas que les mobilités sont du même ordre de grandeur $K_{\mathrm{p}} \simeq K_{\mathrm{n}}$. Des essais du même type sur une solution d'un autre surfactant qui est le sel de chrome de l'acide alkylsalicylique (CrAc) ont fourni les mêmes résultats, la mobilité moyenne étant de $3 \times 10^{-5} \mathrm{~cm}^{2} \mathrm{~V}^{-1} \mathrm{~s}^{-1}$.

4. Discussion. - Nous avons montré que le comportement sous tension continue d'une solution d'Aerosol OT dans le cyclohexane s'explique bien par un phénomène de conduction régie par un équilibre ions-paire d'ions, pour des concentrations comprises entre $10^{-5}$ et $2 \times 10^{-3} \mathrm{Ml}^{-1}$.

Nous avons émis l'hypothèse que cet équilibre est (1) AOT $\rightleftarrows \mathrm{Na}^{+}+\mathrm{B}^{-}$. C'est la réaction se produisant en milieu aqueux; d'autre part, nous ne pouvons 
prendre en considération l'équilibre $2 \mathrm{~S} \rightleftarrows \mathrm{S}^{+}+\mathrm{S}^{-}$(2) que nous avons avancé pour expliquer les variations $\Lambda(C)$ de la conductibilité équivalente des solutions avec leur concentration : une réaction de dissociation bimoléculaire $2 \mathrm{~S} \rightleftarrows \mathrm{S}^{+}+\mathrm{S}^{-}$est peu vraisemblable ; d'autre part, ce type de réaction dans la solution entraînerait la proportionnalité de la densité de courant de saturation $j_{\mathrm{s}}$ avec $(\mathrm{AOT})_{\mathrm{th}}^{2}$, or nous trouvons $j_{\mathrm{s}} \sim(\mathrm{AOT})_{\text {th }}$ préı u par l'équilibre (1).

La réaction équilibrée de dissociation est déplacée par le champ électrique en accord avec le modèle d'Onsager, le temps de relaxation de l'équilibre est égal à la valeur théorique $\varepsilon \rho / 2$, pour un intervalle de concentration $10^{-4}$ à $10^{-2} \mathrm{Ml}^{-1}$; la variation de la constante de vitesse de dissociation $k_{\mathrm{D}}$ avec le champ appliqué est également retrouvée. Nous avons procédé à cette vérification en utilisant une méthode mise au point par Persoons [18] pour étudier l'effet d'un champ électrique sur les solutions d'électrolyte.

D’un point de vue expérimental, il est évident que la détermination de la conductibilité de ces solutions par une mesure du courant continu stationnaire résultant de l'application d'une tension continue d'une centaine de volts est erronée, la conduction n'étant plus ohmique dès $V \simeq 20 \mathrm{~V}$ pour une distance de quelques $\mathrm{mm}$.

En ce qui concerne la conductibilité équivalente $\Lambda$ mesurée en faible tension alternative, nous avons émis l'hypothèse pour expliquer sa quasi-indépendance vis-à-vis de la concentration en AOT, qu'elle était régie par un équilibre $2 \mathrm{~S} \rightleftarrows \mathrm{S}^{+}+\mathrm{S}^{-}$, où $\mathrm{S}$ désigne un agrégat de $m$ molécules. Cette hypothèse est appuyée par la valeur relativement faible de la mobilité des porteurs de charge, et sa non-variation avec la concentration. Le rayon du porteur déduit de la loi de Stokes donne $r=14 \AA$ pour l'Aerosol OT, $28 \AA$ pour $\mathrm{CrAc}$, alors que pour le TIAP nous avons trouvé $6 \AA$. Les porteurs ioniques sont des agrégats. On peut noter que les rayons obtenus n'ont pas de signification physique sûre, seule la variation de cette grandeur d'un électrolyte à l'autre est significative.

Ainsi deux équilibres différents semblent être impliqués pour expliquer le comportement des solutions de surfactants : un équilibre entre paires d'ions et ions pour expliquer la conduction sous champ intense et un équilibre de dissociation d'agrégats

$$
2 \mathrm{~S} \rightleftarrows \mathrm{S}^{+}+\mathrm{S}^{-}
$$

pour expliquer les mesures de conductibilité. Ces faits ne sont pas incompatibles. On peut interpréter l'ensemble des phénomènes observés de la façon suivante.

A l'équilibre thermodynamique, condition réalisée lors de mesures de conductivités au pont en faible tension alternative, il existe dans la solution des agrégats avec une structure privilégiée puisque leur taille est indépendante de la concentration en surfactant. Ceux-ci sont fondamentalement différents des agrégats qui se forment dans des solutions de TBAP ou TIAP dans les hydrocarbures dont l'influence sur la conductibilité a été décrite par Fuoss et Kraus. La réaction globale, résultat d'une succession de réactions, serait $2 \mathrm{~S} \rightleftarrows \mathrm{S}^{+}+\mathrm{S}^{-}$, où $\mathrm{S}$ serait un agrégat à définir par d'autres mesures physiques.

Quand un champ électrique continu existe dans la solution, le système n'est plus en équilibre thermodynamique, et l'état stationnaire de conduction est fixé par la réaction la plus lente qui est la réaction AOT $=\left(\mathrm{Na}^{+}, \mathrm{B}^{-}\right) \rightleftarrows \mathrm{Na}^{+}+\mathrm{B}^{-}$.

La conductibilité des solutions de surfactants dans les hydrocarbures mesurée en tension alternative, les courbes courant-tension continue sont donc maintenant interprétées, certains paramètres comme la taille des agrégats restent à vérifier par d'autres méthodes.

Le comportement sous champs électriques élevés de ces solutions de surfactant est très différent de celui de solutions d'autres composés ionophores aussi du type TBAP, TIAP... que nous avons précédemment étudiés [19]. Dans ces solutions, un courant d'injection d'ions par la cathode est très grand dès $V>100 \mathrm{~V}$ par rapport au courant de conduction dû à la dissociation de l'électrolyte. Dans les̀ solutions de surfactant, aucune injection n'est observée. L'origine de cette injection est une couche chargée dans le liquide, très près de l'électrode, causée par les forces images entre les ions et le métal. De même que les ions au sein de la solution forment des paires d'ions entre eux, les ions proches de l'électrode forment des paires avec leurs images dans le métal. Avec ces électrolytes, on peut dire que les propriétés électriques de ces solutions résultent d'interactions ions-ions, ... (explication par Fuoss et Kraus de $\Lambda(C)$ ) et d'interactions ion-métal de même nature (explication de l'injection par effet Schottky). De même, les propriétés des solutions de surfactant en milieu hydrocarbure résultent d'une agrégation spécifique des molécules dans le volume de la solution (courbe $\Lambda(C)$ ) comme à l'interface (courbe $j(V)$ ou $j(E)$ ).

Enfin, nos résultats rendent improbable l'existence d'une concentration critique micellaire dans une solution d'Aerosol OT en milieu hydrocarbure, comme cela a été annoncé pour une concentration de $10^{-3} \mathrm{Ml}^{-1}$ [20]. En effet, par suite des forces électriques importantes entre les ions en milieu hydrocarbure, il se forme des agrégats même en concentration faible $\left(10^{-5} \mathrm{Ml}^{-1}\right)$.

\section{Bibliographie}

[1] KLInKenberg, A., VAN Der Minne, J. L., Electrostatics in the Petroleum Industry (Elsevier, Amsterdam) 1958

[2] Wennestrom, H., Lindman, B., Phys. Rep. 52 (1979) 74.

[3] Douwes, C., Van der Waarden, M., J. Inst. Petrol. 53 (1967) 237.
[4] Novotny, V., Hopper, M. A., J. Electrochem. Soc. 126 (1979) 925.

[5] Denat, A., Gosse, B., Gosse, J. P., J. Electrostatics 7 (1979) 205. [6] Yasukufu, S., IsE, T., INOUE, Y., Ishioka, Y., I.E.E.E. Trans. Electr. Insul. E-I 12 (1977) 370. 
[7] Fuoss, R. M., Kraus, Ch. A., J. Am. Chem. Soc. 55 (1933) 21, 476, 1019, 2387.

[8] Eicke, H. F., Arnold, V., J. Colloid and Interface Sci. 46 (1974) 101.

[9] Lagües, M., Ober, R., Taupin, C., J. Physique Lett. 39 (1978) L-487.

[10] Thomson, J. J., Thomson, G. P., Conduction of electricity through gases (Cambridge, Univ. Press) 1928.

[11] Silver, M., J. Chem. Phys. 42 (1965) 1011.

[12] Briere, G., Gaspard, F., J. Chim. Phys. 64 (1967) 1071.

[13] Bjerrum, N., Kgl. Danske Vidensk. Selskab. 7 (1926) no 9.
[14] Everaert, J., Persoons, A., Techniques and Applications of fast reactions in solutions, W. J. Gettins, E. Wyn-Jones (Eds) (D. Reidel, Publ. Comp.) 1979, 367.

[15] Onsager, L., J. Chem. Phys. 42 (1965) 1011.

[16] Nakaert, R., De Mayer, M., Hellemans, L., J. Electrostatics 7 (1979) 169.

[17] Allen, A. O., De Haas, M. P., Hummel, A., J. Chem. Phys. 64 (1976) 2587.

[18] Persoons, A. P., J. Phys. Chem. 78 (1974) 1210.

[19] Felici, N., Gosse, J. P., Revue Phys. Appl. 14 (1979) 629.

[20] Muto, S., Meguro, K., Bull. Chem. Soc. Jpn. 46 (1973) 1316 